\title{
Calceolaria ascendens subsp. exigua, comb. et stat. nov. (Calceolariaceae), endémica de la cordillera de la Costa de Chile central
}

\section{Calceolaria ascendens subsp. exigua, comb. et stat. nov. (Calceolariaceae), endemic to Central Chile's Coastal mountain range}

\author{
Nicolás García ${ }^{1,2}$ \\ 1'Department of Biology, University of Florida, Gainesville, FL 32611, USA. \\ ${ }^{2}$ Laboratorio de Flora y Vegetación, Departamento de Silvicultura, Facultad de Ciencias Forestales, Universidad de Chile, \\ Casilla 9206, Santiago, Chile. \\ nicogarcia@ufl.edu
}

\begin{abstract}
The new combination Calceolaria ascendens Lindl. subsp. exigua (Witasek) Nic.García is made based on material collected at Central Chile's Coastal mountain range, around parallel $33^{\circ} \mathrm{S}$. The new subspecies inhabits rock crevices at the high mountain between 1,600 and 2,100 m a.s.l.
\end{abstract}

El complejo Calceolaria ascendens Lindl. está individualizado por la morfología de la corola, que presenta un labio inferior inflado en forma de media esfera, aplanado en la parte superior y cubriendo al labio superior, y lóbulo del elaióforo corto (Ehrhart 2000). La misma autora caracteriza este complejo de taxones y propone un tratamiento integrado por 3 subespecies: $C$. ascendens Lindl. subsp. ascendens, C. ascendens Lindl. subsp. glandulifera (Witasek) C.Ehrh. y C. ascendens Lindl. subsp. pristiphylla (Phil.) C.Ehrh.

Después del estudio de material recolectado en la cordillera de la Costa de Chile central, se ha detectado la existencia de un taxón cuya morfología floral corresponde a la del complejo Calceolaria ascendens y que no concuerda por completo con la descripción de las subespecies tratadas por Ehrhart (2000). En el presente trabajo se realiza una nueva combinación basado en un epíteto ya existente que corresponde al taxón en estudio y éste se compara con las otras entidades infraespecíficas de C. ascendens.

Calceolaria ascendens Lindl. subsp. exigua (Witasek) Nic.García, comb. et stat. nov.

Basiónimo: C. exigua Witasek, Oesterr. Bot. Z. 56: 18. 1906. Tipo: Catemu, Prov. Aconcagua, Philippi s.n. (W; Isotipo: $\mathrm{W})$.

C. miserrima Kraenzl. in Engl., Pflanzenr. Heft 28 (IV. 257C): 119. 1907. C. parvifolia Phil., Anales Univ. Chile 91: 136. 1895, nom. illeg.; non C. parvifolia Weddell
(1857). Tipo: "Locus ignotus”, Philippi s.n. (SGO 055861!; Isotipo: $\mathrm{B} \uparrow, \mathrm{K}, \mathrm{SGO} 043048$ !, US (Foto!, disponible en http://botany.si.edu/types/index.cfm), W, WU).

Arbusto pequeño o subarbusto de hábito postrado y tallos decumbentes a erectos, 8-20 (-30) cm de altura, cubierto completamente por glándulas estipitadas y pelos simples, patentes, cortos ( $\pm 0,1 \mathrm{~mm}$ de largo). Hojas presentes en todo el tallo, opuestas o en fascículos; rugosas, cubiertas en ambos lados por glándulas estipitadas y pelos patentes cortos, ocasionalmente hay presencia de abundantes glándulas sésiles rojas en el envés; nervadura sobresaliente en el envés; contorno oblongo a oblanceolado, 5-25 (33) x 2-10 $\mathrm{mm}$ en la porción más ancha, prolongadas en un pseudopecíolo que abarca $2-10 \mathrm{~mm}$ de largo; borde generalmente entero, ocasionalmente finamente denticulado; ápice agudo. Inflorescencia cimosa, 2-6 cm de largo. Pedicelo floral 5-22 mm de largo, péndulo y curvado cuando el fruto está maduro. Cáliz con sépalos ovados, 4-5 x 2-3 mm; nervadura marcada y sobresaliente; ápice agudo. Corola amarilla; labio inferior inflado en forma de media esfera y arqueado hacia arriba, 12-13 x 8-9 mm; el labio superior es menor que un sépalo y está cubierto por el labio superior, 2 × $3 \mathrm{~mm}$. Anteras 2, subsésiles, 3-4 mm de largo, cubiertas por el labio superior de la corola; filamento 11,2 $\mathrm{mm}$ de largo. Estilo 1-2 mm de largo. Cápsula madura cubierta por glándulas estipitadas, 4-5 x 3-4 mm. Semillas 0,4-0,6 $\mathrm{mm}$ de largo, con ápice apiculado y superficie reticulada (Fig. 1). 


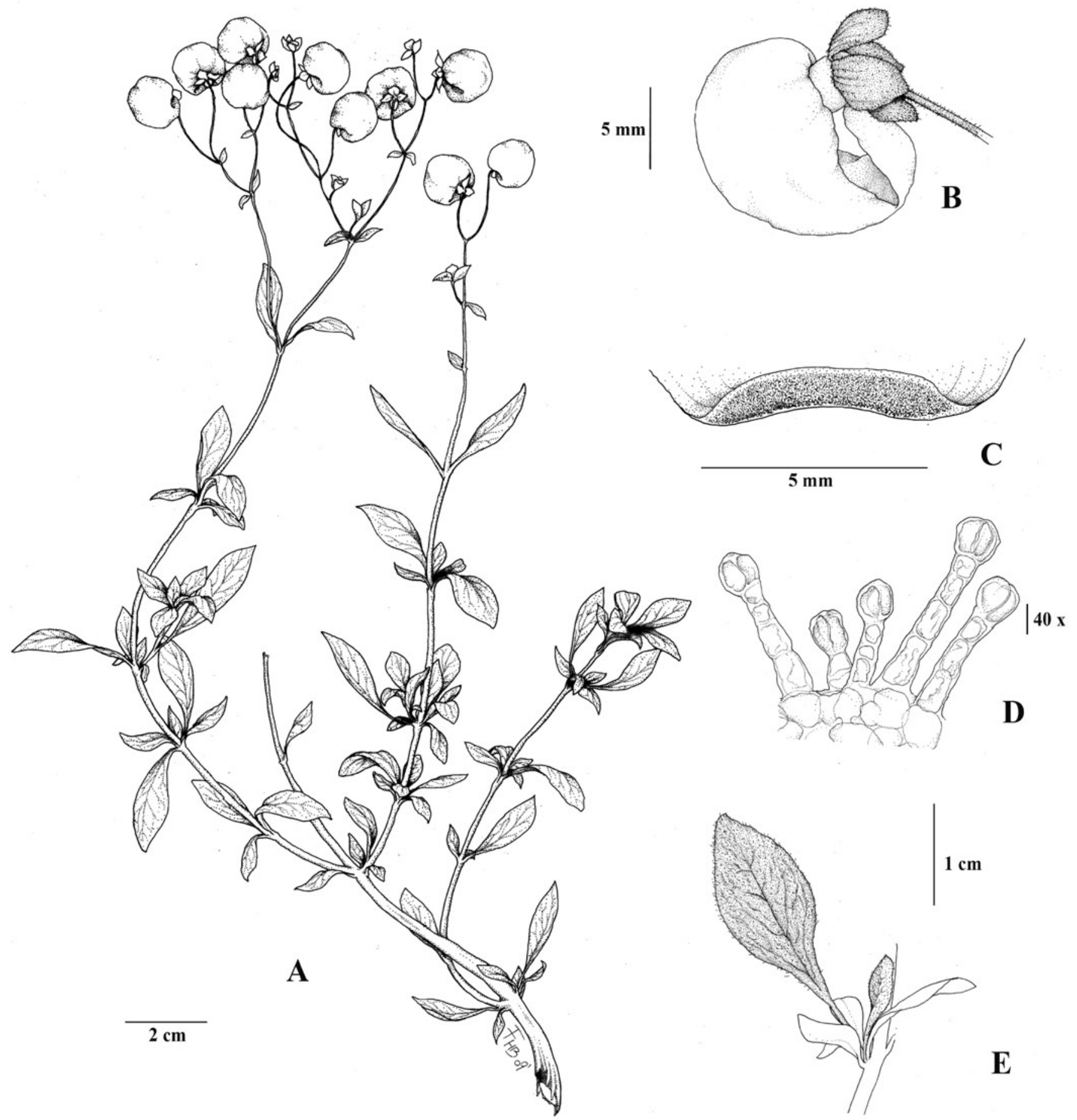

FIguRA 1. Calceolaria ascendens Lindl. subsp. exigua (Witasek) Nic.García: A, rama florífera; B, flor; C, lóbulo del elaióforo; D, pelos oleíferos del elaióforo; E, hoja, vista adaxial (N. García 3891).

FIgURE 1. Calceolaria ascendens Lindl. subsp. exigua (Witasek) Nic.García: A, flowering shoot; B, flower; C, elaiophore-bearing lobe; D, oil-producing hairs of the elaiophore; E, leaf, adaxial view (N. García 3891). 
Se ha examinado el material típico de Calceolaria miserrima Kraenzl. (C. parvifolia Phil.) y se ha constatado que corresponde al taxón en cuestión. Ehrhart (2000) menciona que $C$. miserrima es idéntica a C. exigua Witasek y las trata bajo la sinonimia de $C$. ascendens subsp. glandulifera debido a la escasez de material disponible que fuera atribuible a éstas (i.e. sólo los tipos). En el comentario bajo la subespecie glandulifera, Ehrhart (2000, p. 119) insinúa que ambos taxones fueron descritos sobre materiales de la misma recolección de Rodulfo Philippi, pese a que la procedencia de C. parvifolia Phil. aparece como incierta en las etiquetas de herbario y en el protólogo de la descripción original (Muñoz 1960). El presente autor también opina que este hecho es muy probable, debido al hallazgo en el herbario del Museo Nacional de Historia Natural (SGO) de material recolectado por Philippi procedente de Catemu (localidad donde él mismo recolectó el tipo de C. exigua), en cuyas etiquetas se lee "Calceolaria ascendens var. ?" y que coincide morfológicamente con el tipo de C. miserrima. Adicionalmente, la fecha de recolección de este material (i.e. IX-1860) y las localidades coinciden con lo descrito para la primera excursión de Philippi a la provincia de Aconcagua, donde el taxón en cuestión es tratado como "C. ascendens var. minor" (Philippi 1861), ocasión en la que probablemente también se recolectó el material típico de $C$. miserrima y C. exigua.
Se ha optado por el epíteto "exigua" para realizar la nueva combinación, pese a que el más antiguo es "parvifolia" para no causar una confusión usando un epíteto que es ilegítimo al nivel específico. A pesar que el tipo de C. exigua no pudo ser revisado personalmente o mediante fotografía, la descripción original coincide bien con el material revisado (Witasek 1906).

C. ascendens subsp. exigua se diferencia de las otras subespecies de $C$. ascendens por su hábito de crecimiento bajo, altura en general menor a $30 \mathrm{~cm}$, hojas menores a $3 \mathrm{~cm}$ de longitud y borde de las hojas entero a finamente denticulado. Una fotografía de una rama florecida de este taxón aparece en Luebert et al. (2002, p. 66), donde es tratado como C. ascendens subsp. glandulifera. Por su parte, las otras subespecies son arbustos de ramas ascendentes, altura superior a 50 $\mathrm{cm}$, hojas adultas en general mayores a $5 \mathrm{~cm}$ de longitud y borde notablemente dentado. Las subespecies de $C$. ascendens también se distinguen por la presencia de distintos tipos de indumento. A continuación se presenta una clave para diferenciar las 4 subespecies de este complejo, elaborada a partir del estudio del material citado y de la información disponible en Ehrhart (2000). Esta clave se puede considerar como sustituta de la clave de Ehrhart (2000) desde el punto 22 en adelante.

1. Tallos cubiertos principalmente por pelos glandulosos estipitados; lámina de las hojas cubierta por pelos glandulosos y pelos simples cortos en ambos lados, axilas sin pilosidad lanosa.

2. Ramas decumbentes a ascendentes de menos de $30 \mathrm{~cm}$ de altura; hojas en general de largo menor a $3 \mathrm{~cm}$; lámina de las hojas de borde entero a finamente denticulado. C. ascendens subsp. exigua

2'. Ramas ascendentes de más de $50 \mathrm{~cm}$ de altura; hojas en general de largo mayor a $3 \mathrm{~cm}$ hasta más de $5 \mathrm{~cm}$; lámina de las hojas con borde groseramente dentado. C. ascendens subsp. pristiphylla

1'. Tallos cubiertos principalmente por pelos simples; lámina de las hojas cubierta por pelos simples, axilas barbadas con pelos blanco-lanosos, envés de las hojas jóvenes tomentoso, glabrescente a la madurez.

3. Envés de las hojas con pelos simples y glándulas rojas sésiles dispersas, haz de las hojas densamente cubierto por pelos cortos.

C. ascendens subsp. glandulifera

3'. Envés de las hojas con pelos simples, haz de las hojas con pelos ondulados dispersos.

C. ascendens subsp. ascendens

C. ascendens subsp. exigua ha sido hallada en ambientes rupícolas o roqueríos ubicados en la zona de alta montaña del sistema Aconcagua-Maipo de la cordillera de la Costa de Chile central (García 2006) y en ambientes similares, probablemente a menor altitud, en la vertiente oriental del sistema Cordillera del Melón (Philippi 1861). El rango de altitudes registrado fluctúa entre 1.600 y 2.100 m s.m., y su distribución conocida tiene una extensión de medio grado de latitud geográfica, alrededor del paralelo $33^{\circ} \mathrm{S}$, en las provincias de Petorca, Quillota, Valparaíso y Chacabuco (Fig. 2).
Las poblaciones conocidas de C. ascendens subsp. exigua no presentan algún grado de amenaza de extinción, puesto que no hay desarrollo de infraestructura o actividades humanas recurrentes en los ambientes donde habita, que en general son poco accesibles. Por otro lado, la actividad ganadera es muy esporádica y marginal en su área de distribución. La población del cerro El Roble es la de más fácil acceso, pero está protegida al ser parte del Parque Nacional La Campana y del Santuario de la Naturaleza Cerro El Roble. 


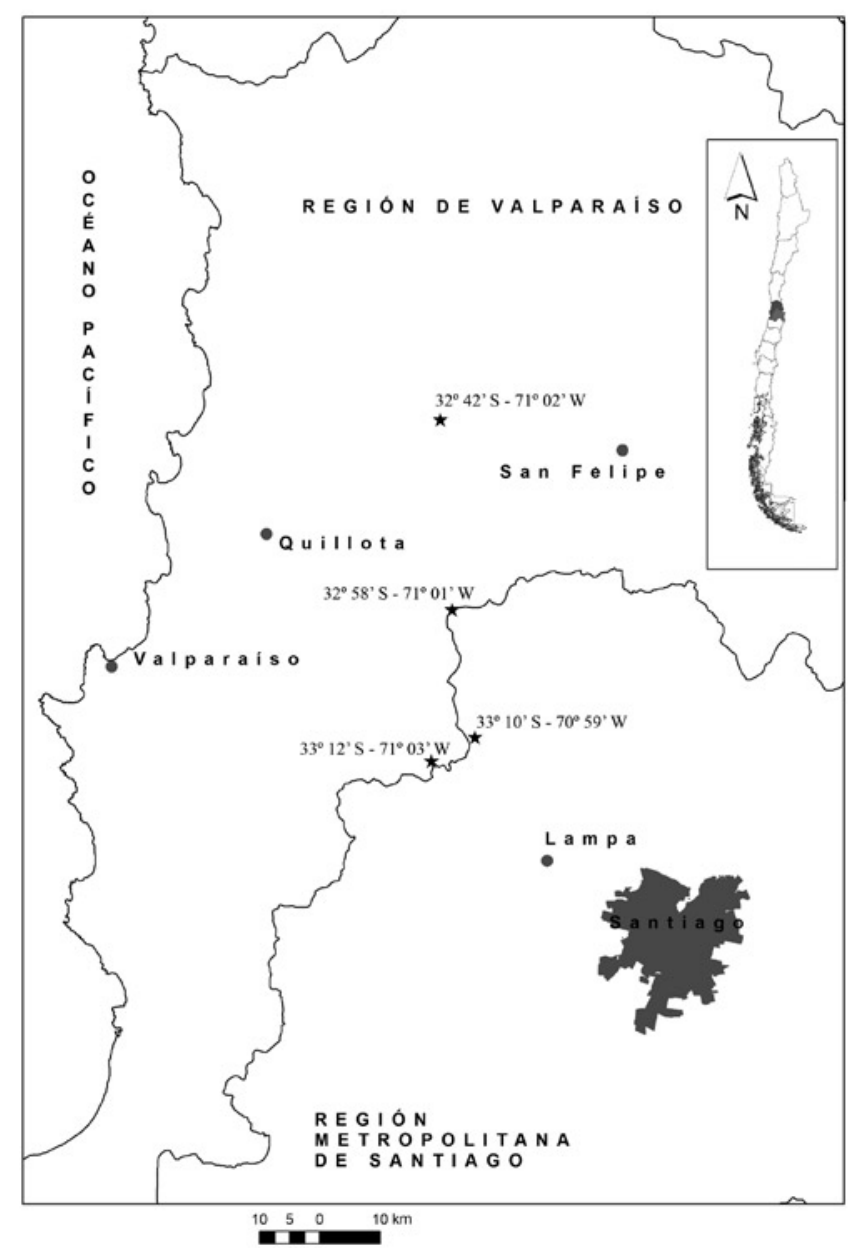

Figura 2. Localidades de colecta de Calceolaria ascendens subsp. exigua.

FIGURE 2. Calceolaria ascendens subsp. exigua collecting localities.

\section{Material ADICIONAL:}

Región de Valparaíso: Prov. Petorca, cajón del Boldo, Catemu (32 $42^{\prime}$ S, $71^{\circ} 02^{\prime}$ W), IX-1860, R.A. Philippi s.n. (SGO 043040); Prov. Petorca, ad cuprifodinas del Salado, Catemu, IX-1860, R.A. Philippi (SGO 056083); Prov. Quillota, cerro El Roble, 22-XI-1972, O. Zöllner 6324 (CONC 80990); Prov. Quillota, cerro El Roble, portezuelo Rauco hacia P.N. La Campana (32 $58^{\circ}$ '43' S, $71^{\circ} 01^{\prime} 08^{\prime}$ '
W), 26-X-2001, A Moreira 578 (SGO 146747); Prov. Quillota, cerro El Roble, roqueríos exposición NO $\left(32^{\circ}\right.$ 58' S, 71º $01^{\prime}$ W), 1.900 m s.m., XII-2002, N. García 3891 (CONC, SGO); Prov. Valparaíso, Colliguay alto, sector

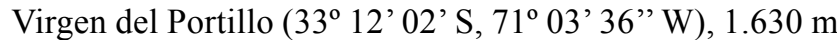
s.m., 01-II-2003, A. Moreira 896 (SGO 150172). Región Metropolitana: Prov. Chacabuco, Altos de Chicauma, línea de cumbres camino al cerro Llaretas de Vizcachas, roqueríos exposición NE $\left(33^{\circ} 09^{\prime} 41^{\prime \prime} \mathrm{S}, 70^{\circ} 59^{\prime} 48^{\prime \prime} \mathrm{W}\right)$, 2.050 m s.m., 21-XII-2004, N. García 631 (CONC, SGO).

\section{AGRADECIMIENTOS}

A Mélica Muñoz y Alicia Marticorena, curadoras de los herbarios SGO y CONC, respectivamente, por facilitar el acceso a dichas colecciones. A Fernanda Herrera por ilustrar el nuevo taxón. A Taryn Fuentes por elaborar la figura de distribución. A Federico Luebert por facilitar datos bibliográficos y traducción del alemán. A Roberto Kiesling por sus consejos nomenclaturales. A dos revisores anónimos por sus comentarios.

\section{BIBLIOGRAFÍA}

Ehrhart, C. 2000. Die Gattung Calceolaria (Scrophulariaceae) in Chile. Bibliotheca Botanica 153: 1-283.

García, N. 2006. Análisis florístico comparativo de la vegetación alto-andina de la cordillera de la Costa y de los Andes de Chile central. Memoria de Título. Facultad de Ciencias Agronómicas, Universidad de Chile, Santiago, Chile. 61 pp.

Luebert, F., M. MuÑoz \& A. Moreira. 2002. Vegetación y flora de La Campana. En: S. Elórtegui \& A. Moreira (eds.), Parque Nacional La Campana. Origen de una Reserva de la Biosfera en Chile central, pp. 36-69. Taller La Era - Fondo de las Américas, Santiago, Chile.

Muñoz, C. 1960. Las especies de plantas descritas por R. A. Philippi en el siglo XIX. Ediciones de la Universidad de Chile, Santiago, Chile. 189 pp.

PhilipPI, R.A. 1861. Botanische Excursion in die Provinz Aconcagua. Botanische Zeitung (Berlin) 19: 377-383, 385-390.

WiTASEK, J. 1906. Die chilenischen Arten der Gattung Calceolaria. Oesterreichische Botanische Zeitschrift 56: 13-20. 